\title{
Regularized Stochastic White Matter Tractography Using Diffusion Tensor MRI
}

\author{
Mats Björnemo ${ }^{1,2}$, Anders Brun ${ }^{1,2}$, Ron Kikinis ${ }^{1}$, and Carl-Fredrik Westin ${ }^{1}$ \\ ${ }^{1}$ Laboratory of Mathematics in Imaging, Brigham and Women's Hospital, \\ Harvard Medical School, Boston MA, USA \\ \{mats, anders, kikinis, westin\} @bwh.harvard.edu \\ ${ }^{2}$ Linköping University, Linköping, Sweden
}

\begin{abstract}
The development of Diffusion Tensor MRI has raised hopes in the neuro-science community for in vivo methods to track fiber paths in the white matter. A number of approaches have been presented, but there are still several essential problems that need to be solved. In this paper a novel fiber propagation model is proposed, based on stochastics and regularization, allowing paths originating in one point to branch and return a probability distribution of possible paths. The proposed method utilizes the principles of a statistical Monte Carlo method called Sequential Importance Sampling and Resampling (SISR).
\end{abstract}

\section{Introduction}

The development of Magnetic Resonance Imaging (MRI) has led to the design of numerous imaging techniques. One of these is Diffusion Tensor MRI (DT-MRI), which measures the motion of hydrogen atoms within water in all three dimensions. In tissue containing a large number of fibers, like skeletal muscle or white brain matter, water tends to diffuse only along the direction of the fibers. The DT-MRI technique has raised hopes in the neuro-science community for a better understanding of the fiber tract anatomy of the human brain. Various methods have been proposed to use DT-MRI data to track nerve fibers and derive connectivity between different parts of the brain in vivo [12, $11,12,13,15,16]$.

A simple and effective method for tracking nerve fibers using DT-MRI is to follow the direction of the maximum diffusion in each voxel, equivalent to the direction of the main eigenvector in each tensor. This method is usually referred to as tracking using the Principal Diffusion Direction (PDD). Although this method is widely spread and used in various ways [4]5], it suffers from some major disadvantages. The connectivity is restricted to a one-to-one mapping between points, not allowing the branching that real fiber tracts may undergo. The PDD tracking also gives the impression of being precise, not taking uncertainty of fiber paths into account in the tracking procedure. Further, the direction of the eigenvector corresponding to the largest eigenvalue is very unstable when in proximity to the generic cases of planar and spherical diffusion [1416.

While there are strong indications that DT-MRI reveals information of the fiber pathways in the brain, it is important to stress the fact that the explicit quantity measured is water diffusion and not fibers. As DT-MRI is a fairly new field of research, many 
studies are yet to be made to compare the measured diffusion tensors to detailed tissue properties important for fiber path inference. However, in contrast to approaches such as solving the diffusion equation [6], it might be important to separate the physical phenomenon of water diffusion from the solution of the tracking problem through the use of a fiber model. In this way a priori knowledge about nerve fibers such as fiber stiffness could be taken into account [12].

In this paper we propose a fiber propagation method that is based on stochastics and regularization, allowing paths originating in one point to branch and return a probability distribution of possible paths. The proposed method utilizes the principles of Sequential Importance Sampling and Resampling (SISR) that belongs to the class of Monte Carlo methods.

\section{Fiber Models}

Inspired by the nomenclature used by Liu et al. [10], single fiber paths will be represented by a sequence $\mathbf{X}=\left(\mathbf{x}_{0}, \mathbf{x}_{1}, \ldots, \mathbf{x}_{N}\right)$, where $\mathbf{x}_{i}$ usually refers to positions in space. Fiber path probability distributions are denoted $\pi(\mathbf{X})$ and the tracking of a fiber path originating from a point $\mathbf{x}_{0}$ give raise to the conditional fiber path distribution $\pi\left(\mathbf{X} \mid \mathbf{x}_{\mathbf{0}}\right)$. The distribution $\pi\left(\mathbf{X} \mid \mathbf{x}_{0}\right)$ assigns a probability to all possible fiber paths originating in $\mathbf{x}_{0}$, which in theory can depend on both the shape of the path, its alignment to the measured diffusion tensor field $\mathbf{D}$ and other prior knowledge. The distribution $\pi_{t}\left(\mathbf{X}_{t}\right)$ will be used to describe the fiber path distribution after $t$ steps of tracking and $\pi_{t}\left(\mathbf{X}_{t-1}\right)$ will denote the probability of the first part of a path $\mathbf{X}_{t-1}$, after $t$ steps of tracking. The tracking is assumed to be finished after $N$ steps and the final distribution is denoted $\pi_{N}(\mathbf{X})$.

The build-up of fiber paths will be sequential, and the direction from the current point, $\mathbf{x}_{t}$, to the next point in the fiber path, $\mathbf{x}_{t+1}$, will be denoted $\dot{\mathbf{x}}_{t}$. The actual distance to the next point on the path will depend on the size of the step, $\Delta t$.

$$
\mathbf{x}_{t+1}=\mathbf{x}_{t}+\dot{\mathbf{x}}_{t} \Delta t
$$

\section{Sequential Importance Sampling and Resampling}

A Monte Carlo method called Sequential Importance Sampling and Resampling (SISR), can be used to calculate an approximation of a probability distribution.

\subsection{Properly Weighted Sample}

In order to work with a distribution $\pi(\mathbf{X})$, it should be represented in some convenient way. One choice of representation is by a set of properly weighted samples [10]. A set of weighted random samples $\left\{\left(\mathbf{X}^{(j)}, w^{(j)}\right)\right\}_{j=1}^{m}$ is called proper with respect to the distribution $\pi(\mathbf{X})$ if for any square integrable function $h(\cdot)$,

$$
E\left[h\left(w^{(j)} \mathbf{X}^{(j)}\right)\right]=c E_{\pi}[h(\mathbf{X})], \text { for } j=1, \ldots, m,
$$



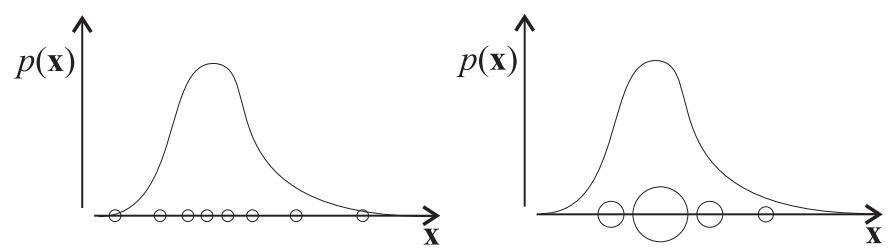

Fig. 1. Left: An unweighted sample representing $p(\mathbf{x})$. Note how the density of samples reflects the distribution. Right: A weighted sample representing $p(\mathbf{x})$. Note how fewer samples can be used to represent the distribution.

where $c$ is a normalizing constant common to all the $m$ samples [10]. Using this definition, it is straight forward to confirm that the expectation $\theta=E_{\pi}[h(\mathbf{X})]$ can be estimated as

$$
\hat{\theta}=\frac{1}{W} \sum_{j=1}^{m} w^{(j)} h\left(\mathbf{X}^{(j)}\right),
$$

where $W$ is a normalizing factor. Drawing for example $\mathbf{X}^{(j)}$ directly from $\pi(\mathbf{X})$ with $w^{(j)}=1$ gives a proper set of samples [10].

The ability to estimate expectations is an important quality for the approximation of a distribution. In fact, every probability distribution, no matter how complicated it is, can be represented to an acceptable degree of accuracy by a set of properly weighted samples from it [10]. However, it might be difficult to perform the actual sampling. One frequently used method to draw random samples from complicated distributions is the Metropolis-Hastings algorithm [7].

By the use of weighted samples the representation can be made more efficient. Figure 1 gives an intuitive understanding of the difference between weighted and unweighted sets of samples. In general, a set of weighted samples can represent a distribution with higher accuracy than a set of equally many non-weighted samples.

\subsection{Importance Sampling}

An often effective way to draw a properly weighted sample $\left\{\left(\mathbf{X}^{(j)}, w^{(j)}\right)\right\}$ from a distribution $\pi(\mathbf{X})$, is to use so called importance sampling. A sample is drawn from a trial distribution $q(\mathbf{X})$ and then assigned a weight $w^{(j)}=\pi(\mathbf{X}) / q(\mathbf{X})$ to make it proper [10].

\subsection{Sequential Build-Up}

In Sequential Importance Sampling a set of properly weighted samples of $\pi(\mathbf{X})$ is built up sequentially, through a series of sample sets of increasing dimension,

$$
\pi_{0}\left(\mathbf{X}_{0}\right), \pi_{1}\left(\mathbf{X}_{1}\right), \ldots, \pi_{N}\left(\mathbf{X}_{N}\right)
$$

smoothly approaching the final distribution so that $\pi_{N}\left(\mathbf{X}_{\mathbf{N}}\right)=\pi(\mathbf{X})$ [10]. Suppose now that we have a set of properly weighted samples $\left\{\left(\mathbf{X}_{t-1}^{(j)}, w_{t-1}^{(j)}\right)\right\}$ representing 
$\pi_{t-1}\left(\mathbf{X}_{t-1}\right)$ and we use a $q_{t}\left(\mathbf{x}_{t} \mid \mathbf{X}_{t-1}\right)$ close or equal to the true $\pi_{t}\left(\mathbf{x}_{t} \mid \mathbf{X}_{t-1}\right)$ to draw a new set of samples $\left\{\left(\mathbf{X}_{t}^{(j)}, w_{t}^{(j)}\right)\right\}$. The weights should then be adjusted according to

$$
w_{t}=w_{t-1} \frac{\pi_{t}\left(\mathbf{X}_{t}\right)}{\pi_{t-1}\left(\mathbf{X}_{t-1}\right) q_{t}\left(\mathbf{x}_{t} \mid \mathbf{X}_{t-1}\right)}=w_{t-1} \frac{\pi_{t}\left(\mathbf{X}_{t-1}\right)}{\pi_{t-1}\left(\mathbf{X}_{t-1}\right)} \frac{\pi_{t}\left(\mathbf{x}_{t} \mid \mathbf{X}_{t-1}\right)}{q_{t}\left(\mathbf{x}_{t} \mid \mathbf{X}_{t-1}\right)}
$$

to represent $\pi_{t}\left(\mathbf{X}_{t}\right)$ [10].

\subsection{Resampling and Reallocating}

Sometimes a representation of a distribution becomes inefficient by having many small weights compared to other dominating large weights. In those cases a more efficient representation can be obtained by pruning away samples with small weights and duplicating samples with large weights by doing a resampling of the distribution. One way of doing this is to generate a new set of properly weighted samples by resampling with replacement from the set, using the weight as a probability for a sample being resampled [10].

\subsection{Related Methods}

Sequential Importance Sampling and Resampling is a method that belongs to the class of population Monte Carlo algorithms [8]. Similar methods are found in for instance control theory and are then often called particle filters. The name has been chosen because samples can be seen as particles in a state space, and the sampling from $q_{t}\left(\mathbf{x}_{t} \mid \mathbf{X}_{t-1}\right)$ can be seen as making the particles walk stochastically along a path in state space. Population Monte Carlo algorithms have also proved to be useful tools in a number of other scientific fields such as statistical physics, quantum mechanics and polymer science [8].

\section{Tracking Using SISR}

Tracking of nerve fibers can benefit from a statistical framework [3]. We will present a model for fiber propagation and relate it to the SISR framework.

\subsection{A Rough Model for Fiber Propagation}

In an attempt to build a more realistic model for fiber propagation, it is assumed that fibers 1) are aligned according to the tensors, 2) do not change direction abruptly in case of spherical and planar tensors, and 3) the uncertainty of fiber propagation increases in planar and spherical tensors.

In the following section, $\mathbf{D}$ will refer to the current tensor $\mathbf{D}\left(\mathbf{x}_{t}\right)$. Also, as the size of the tensor will not matter, the tensor is normed according to its largest eigenvalue, $\lambda_{1}$.

$$
\hat{\mathbf{D}}=\frac{\mathbf{D}}{\lambda_{1}}
$$


The first condition listed above is already accounted for in the PDD tracking method, where the tracking always proceed in the direction of the main eigenvector, $\hat{\mathbf{e}}_{1}$, of the current tensor:

$$
\dot{\mathbf{x}}_{t, \mathrm{PDD}}=\hat{\mathbf{e}}_{1}(\mathbf{D})
$$

To fulfill the second condition, regularization is added to the tracking model. A simple way of doing this is to add a small bias towards the previous tracking direction.

$$
\begin{aligned}
\mathbf{D}_{\text {reg }} & =\left(\hat{\mathbf{D}}+\alpha \dot{\mathbf{x}}_{t-1} \dot{\mathbf{x}}_{t-1}^{T}\right) \\
\dot{\mathbf{x}}_{t} & =\hat{\mathbf{e}}_{1}\left(\mathbf{D}_{\text {reg }}\right)
\end{aligned}
$$

By varying $\alpha$, the bias towards the previous direction can be controlled. This will help stabilize the fiber propagation in case of a spherical tensor, where the main eigenvector might point in any direction before regularization. A larger value of $\alpha$ will smooth the proposed fiber paths to a greater extent.

To incorporate uncertainty in the fiber model, a stochastic part is added perpendicular to the regularized fiber propagation direction. The distribution of the stochastic part is derived from Gaussian noise and transformed using the current tensor to reflect assumption 3), listed before. Thus a linear tensor will result in a small spread.

$$
\begin{aligned}
& \mathbf{e} \in N(\mu, \mathbf{C}), \mu=\left(\begin{array}{lll}
0 & 0 & 0
\end{array}\right)^{T}, \mathbf{C}=\mathbf{I} \\
& \mathbf{r}=\mathbf{D}_{\mathrm{reg}} \mathbf{e}-\left(\mathbf{D}_{\mathrm{reg}} \mathbf{e} \cdot \dot{\mathbf{x}}_{t}\right) \dot{\mathbf{x}}
\end{aligned}
$$

Finally the stochastic part is added to form the complete fiber propagation model.

$$
\begin{aligned}
\dot{\mathbf{x}}_{t}= & \hat{\mathbf{e}}_{1}\left(\mathbf{D}_{\mathrm{reg}}\right)+\mathbf{r}_{p r o j} \beta= \\
& \hat{\mathbf{e}}_{1}\left(\hat{\mathbf{D}}+\alpha \dot{\mathbf{x}}_{t-1} \dot{\mathbf{x}}_{t-1}^{T}\right)+\mathbf{r}_{p r o j} \beta
\end{aligned}
$$

In short, a stabilized propagation direction is calculated and a stochastic part is added. By using different values of $\alpha$ and $\beta$ both the regularization of direction and the stochastic contribution can be varied.

Simulations on synthetic data are shown in figure 2 below. The tensors used in this tracking experiment were disturbed by noise and the overlayed grid is schematic. These simulations show the effect of choosing different values on alpha and beta. For realistic tracking, suitable values on alpha and beta should be estimated and validated.

\subsection{Connections to SISR}

The rough model presented above can be interpreted in the SISR framework, giving it meaning and further guide the search for a good fiber path model. The propagation step of the rough model is viewed as the sequential build-up presented in 3.3 .

- The propagation step of the rough model can be interpreted as sampling from a trial distribution $q_{t}\left(\mathbf{x}_{t} \mid \mathbf{X}_{t-1}\right)=\pi\left(\mathbf{x}_{t} \mid \mathbf{X}_{t-1}\right)$ and assuming that $\pi_{t}\left(\mathbf{X}_{t-1}\right)=$ $\pi_{t-1}\left(\mathbf{X}_{t-1}\right)$. In this way, the rough model fully describes fiber propagation and no further weighting is needed. 

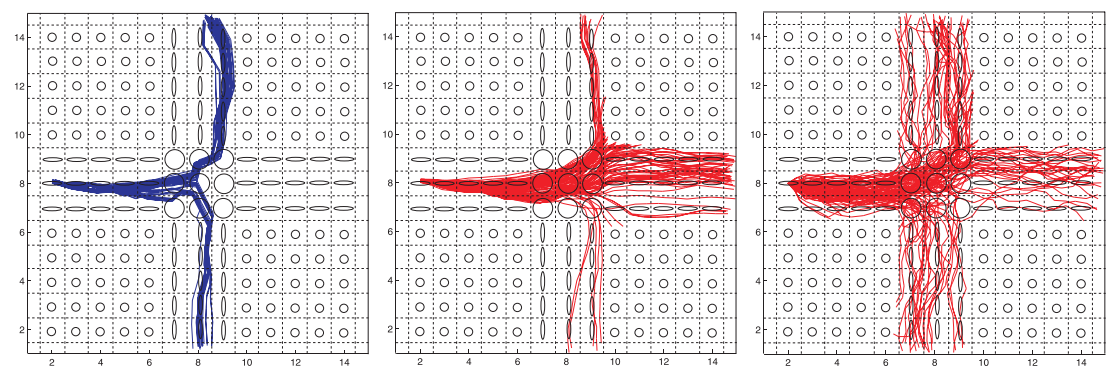

Fig. 2. Left: Normal eigentracking used as a reference (from multiple starting points). Middle: Tracking using a higher value of $\alpha$, resulting in more regularized fiber paths. This supports propagation through spherical tensors. Right: Tractography using a higher value of $\beta$, achieving an increased stochastic spread and a representation of tracking uncertainty in fiber crossings.

- The rough model can be extended by assigning weights to paths by assuming $\pi_{t}\left(\mathbf{X}_{t-1}\right) \neq \pi_{t-1}\left(\mathbf{X}_{t-1}\right)$. Still the conditional path propagation is considered to be fully described by the rough model propagation step as described above, but some compensation is done to for instance to punish some paths. One example is setting the weight to zero or close to zero for a fiber path entering or touching a forbidden area of the brain such as the ventricles.

- To make full use of the SISR framework the propagation step of the rough model should only be considered as the conditional trial distribution close to the real fiber path distribution. This is true importance sampling of the paths and the weight should be adjusted according to equation 4 compensating for the slightly incorrect conditional trial distribution $q_{t}\left(\mathbf{x}_{t} \mid \mathbf{X}_{t-1}\right)$. This is the most general approach, giving a large freedom to choose a realistic fiber model by selecting the weight appropriately.

\section{Results}

The methods mentioned can be simulated using a sequential build-up or sequential sampling algorithm. To ensure a satisfying representation, as many as 100000 samples (particles) were used.

A white matter mask was used to determine when the particles had reached the border between the white and the gray matter, i.e. when to stop the tracking. The mask was created using the EM-MFA segmentation method presented by Kapur et al. [9].

In figure 3 below, the paths of only 200 particles are shown. Notice that the visualized paths should be interpreted as a representation of the probability distribution for 'true' paths, and not as individual 'true' paths themselves.

\section{Discussion}

The method of Sequential Importance Sampling and Resampling has the ability of simulating rich fiber path models. It can take advantage of the full tensor data as opposed to 

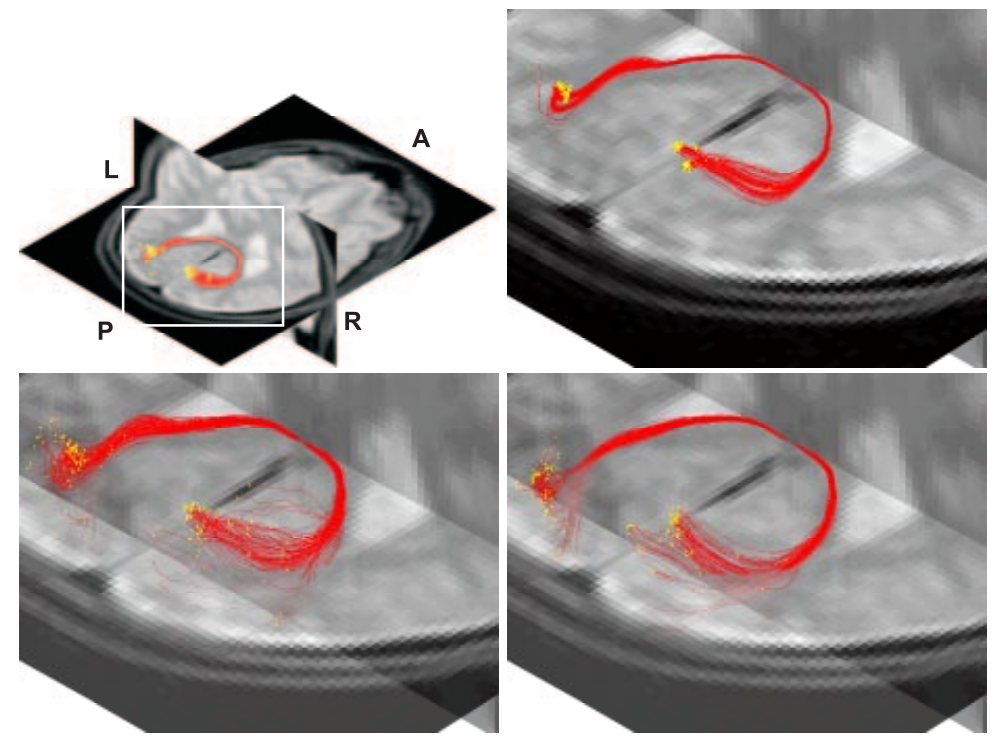

Fig. 3. Top left: Axial and coronal slices indicating the area of the brain where the white matter tractography was performed. Top right: Regularized stochastic tractography, showing one single starting point (at the center of corpus callosum) resulting in multiple end points. Bottom left: Tractography using a higher value of $\beta$, achieving an increased stochastic spread. Bottom right: Tracking using a higher value of $\alpha$, resulting in more regularized fiber paths.

methods based solely on the principal diffusion direction. Though our proposed model depends heavily on the tensor as such, the SISR method is not dependent of the tensor representation. The SISR framework should be easy to adapt to more accurate diffusion measures in the future, a property it is expected to share with other Monte Carlo methods as well. A rich set of fiber models can be used and the theoretical framework provide means for approximately solving a well defined problem up to a chosen level of accuracy.

Despite using a simple model, it has been demonstrated how uncertainty can be taken into account during the tracking procedure. Using a Monte Carlo approach, the tracking paradigm has been extended from a one-to-one mapping to a one-to-many mapping, connecting one starting point to multiple end points in the brain. This could give a different and possibly better view of connectivity in the brain, taking branching of nerve fibers into account.

\section{Acknowledgments}

This work was supported by CIMIT and NIH grant P41-RR13218. 


\section{References}

1. P.J. Basser, S. Pajevic, C. Pierpaoli, J. Duda and A. Aldroubi In Vivo Fiber Tractography Using DT-MRI Data Magnetic Resonance in Medicine, 44:625-632, 2000.

2. P.G. Batchelor, D.L.G. Hill, F. Calamante, and D. Atkinson Study of Connectivity in the Brain Using the Full Diffusion Tensor from MRI IPMI 2001, pp 121-133, Springer-Verlag Berlin Heidelberg, 2001.

3. A. Brun, M. Björnemo, R. Kikinis and C.-F. Westin White Matter Tractography Using Sequential Importance Sampling Proc. of the International Society for Magnetic Resonance Medicine (ISMRM), Honolulu, Hawaii, USA, May 2002.

4. T.E. Conturo, N.F. Lori, T.S. Cull, E. Akbudak, A.Z. Snyder, J. S. Shimony, R. C. McKinstry, H. Burton and M. E. Raichle Tracking neuronal fiber pathways in the living human brain Proceedings of the National Academy of Sciences of the United States of America, 96:1042210427, August, 1999.

5. Z. Ding, J. C. Gore and A. W. Anderson Tracking, Bundling and Quantitatively Characterizing in vivo Neuronal Fiber Pathways Using Diffusion Tensor Magnetic Resonance Imaging Proc. of the International Society for Magnetic Resonance Medicine (ISMRM), 9:1530, Glasgow, Scotland, April 2001.

6. D. Gembris and H. Schumacher and D. Suter Solving the Diffusion Equation for Fiber Tracking in the Living Human Brain Proc. of the International Society for Magnetic Resonance Medicine (ISMRM), 9:1529, Glasgow, Scotland, April 2001.

7. W.R. Gilks, S. Richardson and D.J. Spiegelhalter. Markov Chain Monte Carlo in Practice, Chapman \& Hall, 1996.

8. Y. Iba Population Monte Carlo algorithms Transactions of the Japanese Society for Artificial Intelligence, 2:279:286, 2001.

9. T. Kapur, W.E.L. Grimson, W. M. Wells III and R. Kikinis Enhanced Spatial Priors for Segmentation of Magnetic Resonance Imagery Proceedings of Second International Conference on Medical Image Computing and Computer-assisted Interventions (MICCAI), Cambridge MA, USA, October 1996.

10. J.S. Liu, R. Chen and T. Logvienko. A Theoretical Framework For Sequential Importance Sampling and Resampling Sequential Monte Carlo Methods in Practice, New York, 2001.

11. G.J.M. Parker, C.A.M. Wheeler-Kingshott, and G.J. Barker Distributed Anatomical Brain Connectivity Derived from Diffusion Tensor Imaging IPMI 2001, pp 106-120, SpringerVerlag Berlin Heidelberg, 2001.

12. C. Poupon, C.A. Clark, V. Frouin, J. Regis, I. Bloch, D. Le Bihan, and J.-F. Mangin Regularization of Diffusion-Based Direction Maps for the Tracking of Brain White Matter Fascicles NeuroImage, 12:184-195, 2000.

13. D.S. Tuch, M.R. Wiegell, T.G. Reese, J.W. Belliveau and V.J. Weeden Measuring CorticoCortical Connectivity Matrices with Diffusion Spectrum Imaging Proc. of the International Society for Magnetic Resonance Medicine (ISMRM), 9:502, Glasgow, Scotland, April 2001.

14. C.-F. Westin, S. Peled, H. Gudbjartsson, R. Kikinis and F. A. Jolesz Geometrical Diffusion Measures for MRI from Tensor Basis Analysis Proc. of the International Society for Magnetic Resonance Medicine (ISMRM), Vancouver Canada, April 1997.

15. C.-F. Westin, S. E. Maier, B. Khidir, P. Everett, F. A. Jolesz and R. Kikinis Image Processing for Diffusion Tensor Magnetic Resonance Imaging Proceedings of Second International Conference on Medical Image Computing and Computer-assisted Interventions (MICCAI), 441-452, Cambridge, England, July 1999.

16. C.-F. Westin, S.E. Maier, H. Mamata, A. Nabavi, F.A. Jolesz, R. Kikinis R. Processing and Visualization for Diffusion Tensor MRI Medical Image Analysis, 6(2):93-108, 2002. 